nachgerühmt werden, ob mit Recht, ist noch nicht bewiesen.

Für den Pre Bsch I a m m oder Scheideschlamm sollte man auf Zuckerfabriken eigentlich nicht nach einer nutzbringenden Verwendung suchen, da er ein vorzügliches Düngemittel ist. Leider wird seine Wirkung noch nicht überall geschätzt, und so ist es nicht verwunderlich, daß man nach einer neuen Verwendung sucht. C a y e $n$ glaubt, sie in der Benutzung des Schlammes zur Herstellung von Zement gefunden zu haben. Ein konz. Düngemittel will $\mathrm{L}$ u $\mathrm{t} h$ e $\mathrm{r}$ aus $\mathrm{ihm}$ machen, indem er den getrockneten Schlamm mit Thomasphosphatmehl und schwefelsaurem Ammonium mischt; Ammoniakverluste sollen dabei nicht zu befürchten sein, wie bei der Mischung von schwefelsaurem Ammonium mit Thomasphosphat allein.

\section{Über das Ultramikroskop und seine Anwendung in der Chemie.}

Vortrag gehalten in der Sitzung des Markischen Bezirksvereins am 18. 10. 1905.

Von Privatdozent Dr. med. L. Mrchakurs. (Eingeg. d. 2./4. 1906.)

Meine Herren! Der ehrenvollen Aufforderung Ihres Vorstandes, das Ultramikroskop einem Kreise von Chemikern zu demonstrieren, komme ich hiermit gern nach, weil es nicht schwer ist, vorauszusagen, daß dieser neue optische Apparat manchem von Thnen auch im Fabrikbetriebe bei näherer Kenntnis einmal von Nutzen sein wird. Herr $\mathrm{H}$ ense, der hiesige Vertreter der Thnen allen bekannten Werkstätten von $\mathrm{K}$ a $\mathrm{r} I \mathrm{Z}$ e i B in Jena, hat sich in dankenswerter Weise der Mühe unterzogen, den ganzen komplizierten Apparat hier aufzubauen. Das Ultramikroskop ist ein Apparat, welcher nach einer gewissen Richtung hin die Leistungsfähigkeit des gewöhnlichen Mikroskops von denkbarster Vollkommenheit um ein ganz beträchtliches erweitert. Während die untere Grenze der mit dem Mikroskop sichtbaren Objekte bisher bei etwa $0,2 \mu \mathrm{lag}$, ist es möglich, mit dem Ultramikroskope Objekte zu sehen, deren Durchmesser man kleiner als ein 4000000 stel $\mathrm{mm}(4 \mu \mu)$ schätzen darf.

Der Leistungsfähigkeit des gewöhnlichen Mikroskops ist dadurch eine Grenze gesetzt, daB Objekte mit einem Durchmesser unterhalb eines gewissen Minimum auf keine Weise, durch keine noch so hohe Vergrößerung zur Abbildung gebracht werden können, Man kann sich das so vorstellen, daß die Lichtwellen um die Ränder sehr kleiner Objekte eine Brandung erzeugen, derart, daß die Wellen hinter dem Objekte wieder zusammenschlagen. In dem erleuchteten Gesichtsfeld des Mikroskops erkennt man ein dunkles Objekt dadurch, daß es einen Lichtdefekt in dem Gesichtsfelde erzeugt. So kleine Gegenstände aber, hinter denen die Wellen des Lichtmeeres wieder zusammenschlagen, erzeugen einen solchen Defekt nicht, und sie bleiben unsichtbar, so sehr man auch die Vergrößerung steigern mag. $\mathrm{Helm} \mathrm{holtz}$ und $\mathrm{A} \mathbf{b}$ b e haben berechnet, daß dieses Minimum gleich der halben Wellenlänge des Lichtes ist. Das kurzwelligste, dem Auge sichtbare Licht, das violette Licht, hat eine Wellenlänge von ungefähr $0,4 \mu$. Alle Objekte, deren Durchmesser kleiner als die Hälfte dieses Wertes ist, bleiben uns bei noch so hoher Vergrößerung unsichtbar. Arbeitet man mit ultraviolettem Licht, welches eine noch etwas kürzere Wellenlänge hat, so wird das Auflösungsvermögen des Mikroskops noch um ein weniges erhöht. Da das ultraviolette Licht nicht direkt für das Auge sichtbar ist, so hat $\mathrm{K}$ ö h l e r einen sehr ingeniösen Apparat konstruiert, bei dem das Auge durch die photographische Platte ersetzt wird. Aber auch die Leistungsfähigkeit dieses Apparates erreicht sehr bald eine Grenze.

Das Ultramikroskop ist nach einem ganz anderen Prinzip konstruiert. Die Geschichte seiner Entstehung ist die folgende.

$\mathrm{R}$ i c h a r d Z s i g m o n d y beschäftigte sich mit den Eigenschaften des Goldrubinglases. Wenn man etwas metallisches Gold mit Glas zusammenschmilzt, so hat bei bestimmten Bedingungen der Abkühlung das erstarrte Glas eine rubinrote Farbe bei völlig erhaltener Durchsichtigkeit. Wenn man mittels einer Sammellinse Rubinglas mit Sonnenstrahlen beleuchtet, so sieht man einen hellen Lichtkegel, gerade so, wie wenn man ein von Staub getrübtes Wasser in der gleichen Weise beleuchtet, oder wenn die Sonne die Staubteilchen der Luft im Zimmer zeigt. Schon F a r a d a y machte deshalb die Annahme, daß die rote Farbe des Rubinglases auf dem Vorhandensein allerfeinster Goldstäubchen im Glase beruhe. Wenn man aber ein Stück Rubin. glais unter das Mikroskop legt, so sieht man nichts von den vermuteten Goldteilchen. Man mußte daraus schließen, daß die Goldteilchen einen kleineren Durchmesser haben, als er dem oben erwähnten $\mathrm{Helmholtz} \cdot \mathrm{Ab}$ beschen Grenzwerte entspricht.

Nun war es aber schon durch Untersuchungen von $\mathbf{F i z e a} \mathbf{u}$ und $\mathbf{A} \mathbf{m}$ bron $\mathbf{n}$ bekannt, daß man unter anderen Beleuchtungsbedingungen als sie am Mikroskop gegeben sind, noch von viel kleineren Gegenständen einen Lichteindruck erhalten konnte. Wenn man durch einen in seiner Entstehung Ihnen ja bekannten Silberspiegel in die Sonne blickt, so erkennt man noch Spalten von viel geringerer Breite, als jenes Minimum. Ebenso sieht man auf dem dunklen Untergrunde des Himmels die Fixsterne, deren Durchmesser so klein ist, daß auch die stärksten Fernrohre sie nur als Punkt zeigen. Hier handelt es sich auch nicht um die, ,ähnliche Abbildung", sondern nur um einen Lichteindruck. Wenn die Fixsterne dreieckig wären, so würden sie in unserem Auge den gleichen Eindruck erzeugen, als wenn sie rund sind.

Wenn es sich also nur um die Erzeugung eines Lichtpunktes handelt, so kann man noch Objekte von unglaublicher Kleinheit sehen, wenn sie nur selbstleuchtend auf dunklem Untergrunde stehen. Das $\mathrm{H} \mathrm{e} \mathrm{I} \mathrm{m} \mathrm{h}$ ol t z - A b b e sche Gesetz gilt nur, wo es sich um ähnliche Abbildung handelt, es ist aber keine Schranke, wo es sich um den bloßen Lichteindruck eines kleinen leuchtenden Objektes, wohl gemerkt, aber auf dunklem Untergrunde handelt.

$Z$ s i g m o n d y überlegte sich also: Wenn es gelänge, die Goldteilchen im Glase selbstleuchtend 
zu machen, so müßte man sie sehen können. Es ist nun ganz leicht, die Goldteilchen zum Leuchten zu bringen. Sie leuchten ja, wie gesagt, wenn man sie durch eine Sammellinse mit Sonnenlicht beleuchtet, nur darf man nicht in die Richtung der Lichtstrahlen blicken, sondern muB den Lichtkegel von der Seite her betrachten. Das hatte man aber im Mikroskop noch nie versueht, denn hier blickt man ja absichtlich immer in die Richtung der Beleuchtungsquelle. $Z$ sig mond y betrachtete nun aber einmal den mit der Linse entworfenen Lichtkegel von der Seite, $d$. h. er lie $B$ die Sonnenstrahlen in der Ebene des Objekttisches einfallen, senkrecht zur Tubusrichtung des Mikroskops, und betrachtete den Lichtkegel mit dem Mikroskop von oben her. Un d da sah er die so lange vermißten Goldteilchen, wie zahllose stern chen a uf unklem Untergrund leucht e nd; er brauchte dazu nicht einmal besonders starke Vergrößerung. Das ist das ganze Geheimnis des Ultramikroskops. des Mikroskops angebracht ist und in Fig. 2 noch einmal deutlicher zu sehen ist. Diese Küvette wird durch eine aus der Abbildung leicht ersichtliche Vorrichtung mit der za untersuchenden Flüssigkeit gefüllt. Will man Goldrubinglas untersuchen, so braucht man an Stelle der Küvette nur einen entsprechenden Block aus Rubinglas zu stellen. So befindet sich also ein intensiver Strahlenkegel in der Küvette bzw. dem Block, welchen man von oben her mit dem Mikroskop betrachtet. Während also die Richtung der Lichtstrahlen horizontal geht, blickt man durch das Mikroskop in vertikaler Richtung.

Mit dieser Einrichtung kann man Flüssigkeiten oder genau passend abgeschliffene Glasblöcke untersuchen. Um den Apparat auch für andere, zwischen Objektträger und Deckglas liegende Objekte benutzbar zu machen, hat ihm $\mathrm{S}$ i ed e n t o $\mathrm{p}$ f noch eine zweite Form gegeben welche aus Abbildung 3 ersichtlich ist. Das Mikroskop liegt hier horizontal, und es wird durch ein ganz anderes Prinzip verhindert, daß die von der Bogenlampe kommenden

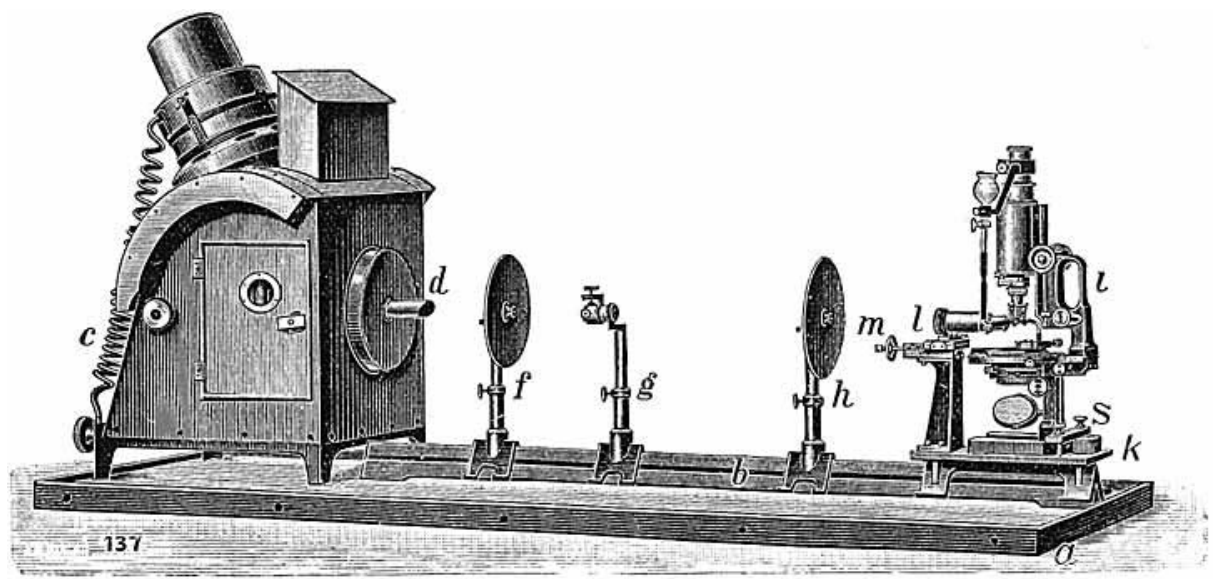

Abb. 1.

Um aber aus diesem Prinzip ein wirklich brauchbares optisches Instrument zu machen, bedurfte es noch zahlreicher optischer Berechnungen und Konstruktionen, welche der Physiker K a r l S i ed e nto pf im Laboratorium der Zeißschen Werke in geradezu vollkommener Weise gelöst hat. In der Abbildung 1 sehen Sie, welche Form dieser Apparat durch Si den t o p f erhalten hat. $c$ stellt die Beleuchtungsquelle, eine elektrische Bogenlampe dar. Die durch die schornsteinartige Blende $\mathrm{d}$ gelangenden Lichtstrahlen werden zunächst durch die Sammellinse $f$ zur Konvergenz gebracht. An der Vereinigungsstelle der Strahlen befindet sich ein in seiner Weite regulierbarer Präzisionsspalt g, durch

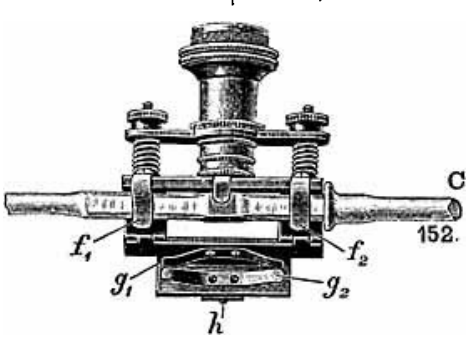

Abb. 2
Strahlen in das Mikroskop gelangen. Vor dem Objektiv befindet sich nämlich an Stelle des $\mathrm{A} \mathrm{b} \mathrm{b} \mathrm{e-}$ schen Kondensors ein Linsensystem, welches die Lichtstrahlen derartig zurKonvergenz bringt, daß die Breite des Lichtkegels da, wo sie auf das Objektiv trifft, nur das Zentrum der Linse bedeckt, und dieses Zentrum ist durch eine die Mitte der Linse ausfüllende Schwärzung undurchsichtig gemacht. So gelangt also von der Lichtquelle kein Strahl in das Objektiv. Das Objekt wird in gewöhnlicher Weise zwischen $\mathrm{Ob}$ jektträger und Deckglas untersucht. Von dem Objekt können aber nur diejengen Strahlen in das Objektiv gelangen, welche durch Betgung die seitlichen, nicht abden man nach Belieben ein breiteres oder schmaleres Strahlenbündel herausschneiden kann. Von diesem leuchtenden Spalt wird mittels der Sammellinse $h$ ein verkleinertes, umgekehrtes Bild dicht vor l entworfen, und von diesem wird wiederum ein Strahlenkegel durch die Sammellinse 1 in der Küvette C erzeugt, welche unterhalb des Objektivs geblendeten Teile der Linse treffen. Hier wird also durch ein ganz anderes Prinzip dasselbe erreicht, daß nämlich von der Lichtquelle direkt kein Strahl in das Auge des Beobachters gelangt, sondern nur dasjenige Licht, welches vom Objekt abgebeugt wird. Durch eine einfache Wechselvorrichtung kann man diesen Kondensor gegen einen gewöhnlichen $\mathrm{A}$ b b e schen 
Kondensor auswechseln, welcher auch die seitlichen durchsichtigen Partien der Linse erleuchtet, und dadurch das Ultramikroskop in ein gewöhnliches Mikroskop verwandelt. Diese Einrichtung des zen tral abgeblendeten Objektivs stellt die vollendetere Ausführung eines schon früher bekannten Prinzips der sogenannten $D$ u $\mathbf{n} k$ e l f e l d bel e u c h t ung dar, welche allerdings an Leistungsfähigkeit sich nicht im entferntesten mit der neuen Einrichtung messen kann und daher noch keines der Resultate gezeitigt hatte, welche das Ultramikroskop heute ergibt.

Welcher Anwendung ist nundas U ltramikrosk o p ähig ? Das größte In. teresse für den Chemiker bildet zunächst das Studium der kolloidalen Lösungen, wie Lösungen von Eiweiß, Glykogen, manchen Farbstoffen und kolloidale Metallösungen. Die Lösungen zeigen schon mit bloßem Auge niemals die vollkommene Klarheit wie die Lösungen kristalloider Körper. Im durchfallenden Licht sind sie - sofern nicht eine intensive Färbung daran hinderlich ist - scheinbar
Teilchen sich zu Boden senken. Die Farbe der Goldlösung ist im durchfallenden Licht rot, im Ultramikroskop erscheinen die Teilchen grünlich. Das kommt daher, daß gerade diejenigen Lichtstrahlen, für welche die Goldteilchen durchlässig sind, sich in dem abgebeugten Licht nicht vorfinden. So erscheint im Ultramikroskop die Farbe der sichtbaren Teilchen häufig ungefähr komplementär zu der gewöhnlichen Farbe im durchfallenden Licht. Wenn durch Zusatz von Elektrolyten die Goldlösung koaguliert wird, so verschmelzen die kleinen Teilchen zu größeren, helleren, weniger zahlreichen, bis sie schließlich mikroskopische Größe erreichen und zu Boden fallen.

Die G r ö B e der schwebenden Goldteilchen ist von $S$ i edentopf und $Z s i g m$ ond auf die Weise bestimmt worden, daß sic in einem sehr kleinen, genau ausgemessenen Volumen, welches einen Bruchteil des mikroskopischen Gesichtsfeldes bildet, die Menge der Teilchen durch Zühlung bestimmten und andererseits durch chemische Analysen den Goldgehalt ermittelten. So konnten sie rechnerisch

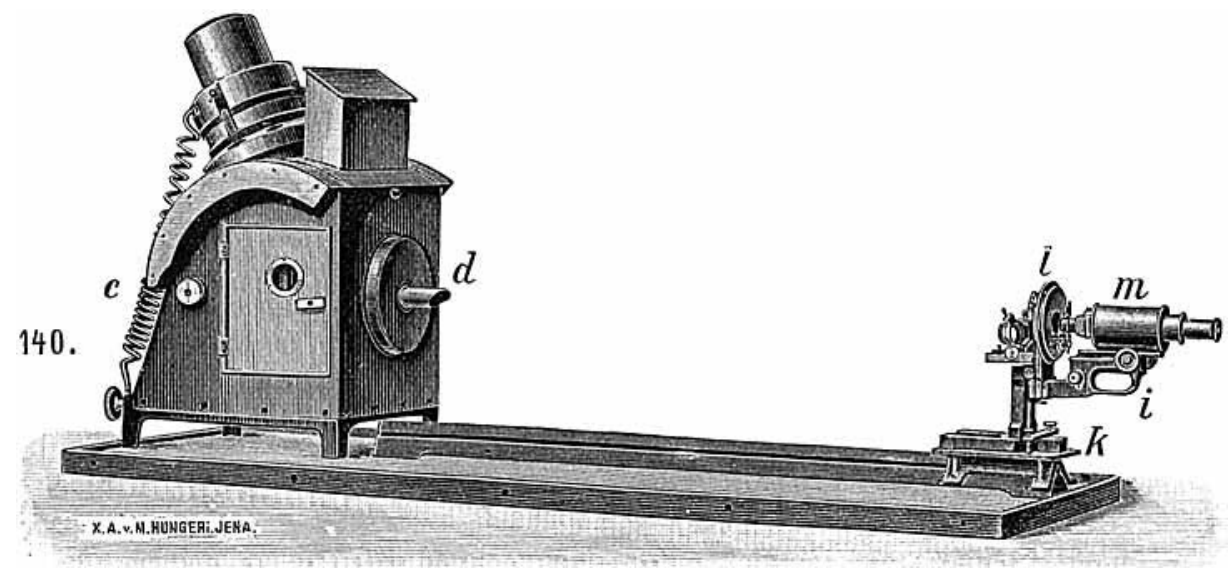

Abb. 3.

völlig durchsichtig, aber bei Beleuchtung mit einer Sammellinse leuchtet der Lichtkegel in ihnen hell auf, und das von dem Lichtkegel ausgesendete Licht erweist sich als polarisiert. Schon daraus mußte man schließen, daß das Licht von kleinsten schwebenden Partikeln reflektiert worde, und diese Vermutung ist durch das Ultramikroskop bestätigt worden. Von den kolloidalen Metallösungen ist von $\mathrm{S}$ i e den topf und $\mathrm{Zsigmondy}$ zuerst die kolloidale Goldlösung studiert worden. Im Ultramikroskop verhält sie sich wie Goldrubinglas; zahllose Sternchen leuchten auf dunklem Untergrund. Aber sie stehen nicht still, wie im Rubinglas, son. dern zeigen eine sehr lebhafte Eigenbewegung von doppelter Natur. Zunächst bewegen sie sich lebhaft oszillatorisch, sie tanzen hin und her; außerdem aber zeigen sie auch eine translatorische Bewegung, welche meist im Zickzack vor sich geht. Diese Bewegung erinnert an die sogenannte $\mathrm{Br}$ r w n sche Molekularbewegung, welche feine Körnchen, wie Aufschwemmungen von chinesischer Tusche, im gewöhnlichen Mikroskop zeigen. Diese Bewegung ist wohl als das Produkt der zwischen den Körnchen herrschenden abstoßenden Kräfte zu betrachten, denselben Kräften, welche es verhindern, daß die leicht zu einer Größenbestimmung der Goldteilchen gelangen. Es zeigte sich da, wie es auch anzunehmen war. daß die Größe bei verschiedenen Proben von Goldlösung erheblich schwankte. Die kleinsten Werte waren $6 \mu \mu$ (Millionstelmillimeter) Seitenlänge, wenn die Teilchen als Würfel gedacht werden, die größten 30-40 $\mu \mu$. Wenn die Teilchen noch größer wurden, so war die Lösung nicht mehr haltbar, und die Teilchen senkten sich bald zu Boden, wenn sie nicht durch ein sogenanntes Schutzkolloid, z. B. Leim, künstlich in der Schwebe gehalten wurden.

Im übrigen ist die Annahme, daß die Goldteilchen Würfelform oder überhaupt eine radiär symmetrisches Form haben, mit Sicherheit unzutreffend. Alles spricht dafür, daß sie vielmehr als Plättchen oder Nädelchen gedacht werden müssen. Aber direkt gibt, wie gesagt, das Ultramikroskop keine Vorstellung von der wahren Form. Besonders lehrreich in dieser Beziehung ist ein Objekt, welches S i edont opf untersucht hat, nämlich die gefärbten Steinsalzkristalle. Steinsalz findet sich mit. unter in der Natur gefärbt, und künstlich läßt es sich färben, wenn man es Dämpfen von Natrium aussetzt oder mit Elektrizität, Röntgenstrahlen 
oder dgl. eine Ionisation in dem Kristall hervorruft, welche die Bildung von freiem Natrium veranlassen kann. Es war daher wahrscheinlich, daß die Färbung auf dem Vorhandensein feinster Natriumteilchen beruhte. Im Ultramikroskop sah $\mathbf{S}$ i e d e $\mathbf{n}$ t o $\mathrm{p} f$ in der Tat in bestimmten Anordnungen in den Steinsalzkristallen feinsteKörnchen, welche einen ähnlichen Eindruck machten wie die Goldteilchen im Rubinglas. Er konnte, wie auch sonst, konstatieren, daß das im Ultramikroskop sichtbare Bild dieser Teilchen polarisiertes Licht aussendete. Aber die Polarisationsebene war nicht immer die gleiche. Daraus konnte er, aus theoretisch-optischen Gründen, schließen, daß die Teilchen nach verschiedenen Dimensionen einen verschiedenen Durchmesser haben müssen, und daß man sie sich als Nädelchen, nicht aber als Kugeln oder Würfel vorzustellen habe.

Ein zweites Objekt für das Ultramikroskop bieten die Lösungen der Farbstoffe. Siedepunktsbestimmungen und dgl. physikalische Methoden haben schon früher oft ein viel zu hohes Molekulargewicht bei manchen Farblösungen ergeben, und es war daher naheliegend, anzunehmen, daß manche Farbstofflösungen nicht echte, sondern kolloidale Lösungen darstellten. Die näheren Untersuchungen, welche an einem Beispiel (Fluorescein) schon von $\mathrm{Si}$ ed e n t o p und $\mathrm{Zsigmondy}$ begonnen, dann von $R$ a e $h l m$ a $n n$ sowie von mir näher ausgeführt wurden, haben ergeben, daß man ihrem optischen Verhalten nach die Farbstoffe in verschiedene Klassen einteilen kann.

Zunächst gibt es Farbstofflösungen, welche sich genau so verhalten wie eine Goldlösung: im Ultramikroskop sieht man die Farbstoffkömohen meist in ihrer Komplementärfarbe auf dunklem Untergrund. So verhalten sich z. B. vor allom das , lösliche Berliner Blau" ( $R$ a e hlmann), dann auch viele hochmolekulare organische Farbstoffe, wie das Violettschwarz, Indulin, Nigrosin. In sehr verdünnten Lösungen von Berliner Blau sieht man äußerst dicht gedrängte, kupferfarbene Lichtpünktchen, zwischen denen das Gesichtsfeld dunkel ist. Auf der anderen Seite gibt es wiederum Farblösungen, die sich selbst im Ultramikroskop nicht in Körnchen auflösen lassen. Hierher gehören alle fluoreszierenden Farbstoffe. Im Ultramikroskop sieht man natürlich nicht die Eigenfarbe des betreffenden Farbstoffes, sondern die Fluoreszenzfarbe, und diese völlig diffus, nicht in Körnchen auflösbar, wie eine gefärbte Milchstraße.

Das ist zunächst sehr auffällig. Fluoreszierende Lösungen haben eine große Ähnlichkeit mit getrübten Flüssigkeiten; in beiden leuchtet der Strahlenkegel einer Sammellinse hell auf, und man hätte erwarten sollen, daß auch das Ultramikroskop für beide ähnliche Bilder ergeben sollte. Das ist aber nicht der Fall. Und schließlich besteht ja auch ein sehr wesentlicher Unterschied zwischen einer getrübten und einer fluoreszierenden Flüssigkeit. Der Strahlenkegel in der ersteren sendet polarisiertes, der Strahlenkegel in der letzteren nicht polarisiertes Licht aus. Das beweist schon, daß die Fluoreszenz auf ganz andere Weise entsteht als die Trübung. Die Teilchen, an denen die Fluoreszenz entsteht, sind offenbar noch von einer viel geringeren Größenordnung als die Teilchen einer kolloidalen Metallösung.
Drittens aber gibt es Farbstoffe, die gerade in der Mitte zwischen diesen beiden Gruppen stehen; sie zeigen zwar im Ultramikroskop einen in Körnchen auflösbaren Lichtkegel, aber die Körnchen sind relativ so spärlich, daß sie nicht den gesamten Farb. stoffgehalt der Lösung darstellen können. Dahin gehört z. B. Fuchsin und Methylviolett. In einerFuchsinlösung 1 : 1000 sieht man noch verhältnismäßig wenig Körnchen. Der Farbstoff ist hier in zwei Phasen in dem wässerigen Medium enthalten, erstens als echte homogene, optisch nicht auflösbare Lösung, zweitens in körnig suspendiertem Zustand. Die beiden Phasen stehen in einem Gleichgewichtszustand zueinander; durch verschiedene Zusätze kann man die körnige Phase vermehren, z. B. durch Zusatz von Kochsalz, bis schließlich bei einem noch höheren Kochsalzgehalt die „Aussalzung" des Farbstoffs beginnt. In der histologischen 'Technik benutzt man seit $\mathbf{E}$ hrli o h als Zusatz zu vielen Anilinfarbstoffen zur Erhöhung der Färbekraft einige organische Substanzen, wie Anilinwasser und dgl. Wenn man nun ultramikroskopisch untersucht; welchen Einfluß diese Zusatzstoffe auf eine Fuchsinlösung haben, so sieht man, daß sie die körnige Phase der wässerigen Lösungen erheblich vermehren. Damit Hand in Hand geht die Tatsache, daß solche Farblösungen beim längeren Aufbewahren leicht Niederschläge geben. Es wäre aber nicht richtig, sich vorzustellen, daß die ultramikroskopisch sichtbaren Farbstoffkörnchen es sind, welche von der Faser adsorbiert würden und dadurch das Färbevermögen erzeugten; es spricht vielmehr alles dafür, - näher kann ich hier nicht darauf eingehen -, daß im Gegenteil das Färbevermögen nur an den ultramikroskopisch $\mathbf{n}$ i c $\mathbf{h}$ t auflösbaren, also wirklich gelösten Anteil des Farbstoffes haftet. Das Färbevermögen einerseits, und die Kondensation des Farbstoffs zu ultramikroskopischen Kömchen, beides ist nur derselbe Ausdruck der gleichen Grunderscheinung: der Leichtigkeit, mit welcher ein solcher Farbstoff seiner wässerigen Lösung entrissen werden kann.

Noch einige Beispiele aus der Farbstoffchemie möchte ich geben, welche die Leistungsfähigkeit des Ultramikroskops in das rechte Licht zu setzen geeignet sind.

Wenn man eine äußerst verdünnte, wässerige Lösung, z. B. von Thionin ( $\mathrm{L}$ a $\mathrm{u} \mathrm{t} \mathrm{h}$ schem Violett), welehe blauviolett ist, mit $\mathrm{NaOH}$ versetzt, so entsteht die freie Thioninbase mit roter Farbe, welche eigentlich in Wasser unlöslich ist. Aus sehr verdünnten Lösungen entsteht aber doch kein Niederschlag der Base, sondern eine scheinbar homogene, tote Lösung, welche im Dunkeln sich sehr lange hält (durch Sonnenlicht allerdings rasch zur Koagulation gebracht werden kann). Wenn man jedoch diese rote Lösung im Ultramikroskop betrachtet, so sieht man, daß der gesamte Farbstoff nicht als wirkliche Lösung, sondern als Suspension feinster, ultramikroskopischer Partikel vorhanden ist. Die blaue Thioninlösung dagegen zeigt im Ultramikroskop gar keine Körnchen, sondern nur eine völlig homogene, nicht polarisierte rote Fluoreszenz; das Thionin, in Form seiner Salze, bildet also in Wasser eine echte Lösung, die Thioninbase dagegen eine ultramikroskopische Suspension. Wenn man die Thioninbase dagegen in Toluol löst, so zeigt das 
Ultramikroskop wiederum nur eine Fluoreszenz, keine körnige Struktur.

Somit ist durch das Ultramikroskop bestätigt worden, daß die Farbstoffe einen gewissen Übergang von den kristalloiden zu den kolloidalen Stoffen bilden; sie bilden zum Teil wirkliche, echte Lösungen, neigen aber sehr dazu, unter vielen $\mathrm{Be}$ dingungen in feinste Suspensionen überzugehen.

Ein anderes Gebiet umfassen die E i w e i B körper. Die Lösungen der Eiweißkörper sind schon ohne optische Hilfsmittel als opak zu erken. nen, aber das Mikroskop konnte die Ursache dieser Trübung bisher nicht aufdecken. Das Ultramikroskop gibt aber auch hier näheren AufschluB. Betrachtet man eine stark verdünnte Eiweißlösung (z. B. verdünntes Blutserum) ultramikroskopisch, so erscheint das Gesichtsfeld von feinsten, schwebenden leuchtenden Punkten übersät (Abb. 4). Betrachtet man das Blutserum im unverdiünten $\mathrm{Zu}$ stand, so liegen die Teilchen so dicht beieinander, daß sie nicht einzeln unterschieden werden können, sondern das ganze Gesichtsfeld einen diffusenLichtschein gibt. Die einzelnen Körnchen liegen hier so dicht beieinander, daß das Mikroskop den Nebel nicht auflösen kann. Was beim gewöhnlichen Mikroskop für die Größe des $\mathrm{Ob}$ jekts selbst gilt, das gilt hier für den $\mathrm{Ab}$. stand der einzelnen Teilchen. Sobald dieser kleiner ist, als der H e l m h o l t z - A b besche Grenzwert, so bringt das Mikroskop die Teile nicht einzeln zur Anschauung. Dies geschicht erst bei genügender Verdünnung der Eiweißlösung. Da nun diese Teilchen außerordentlich klein sind und sich schon den Dimensionen nähern, die wir den größeren Molekülen zuschreiben dürfen, so vermutete man anfangs, daß vielleicht die sichtbaren Eiweißkörnchen die Eiweißmoleküle seien. Jedoch hat sich das nicht bestätigt. Dafür sprechen mehrere Beobachtungen. $R$ a $e \mathbf{h} \mathbf{l} \mathbf{m}$ an $\mathbf{n}$ beobachtete folgendes. Wenn man eine Eiweißlösung so stark verdünnt, daß sie im Gesichtsfeld nur noch einige wenige Körnchen zeigt, so tritt nach dem Kochen dieser Lösung eine erhebliche Vermehrung der Körnchen ein. Ich konnte ferner zeigen, daß es einen großen Unterschied macht, ob man Blutserum mit destilliertem Wasser oder mit $0,9 \%$ Kochsalzlösung verdünnt. In destilliertem Wasser erseheinen die Körnchen viel zahlreicher als in Kochsalzlösung. Da nun das Kochen durch Koagulation des Eiweißes, das destillierte Wasser durch Ausfällen des Globulins einen Niederschlag erzeugt, so ergikt sich, daß das Auftreten der Körnchenvermehrung nichts weiter ist, als eine Vorstufe der Niederschlagsbildung. Man hat sich also, wie bei der einen Gruppe von Farb- stoffen, die Sache so vorzustellen, daß das Eiweiß in zwei Phasen im Wasser vorhanden ist, einer echten Lösung, von der auch das Ultramikroskop nichts zeigt, und zweitens in Form einer ultramikroskopischen Suspension. Wieviel von der einen und der anderen Form vorhanden ist, hängt jedesmal von vielen Faktoren ab, z. B. vom Salzgehalt der Lösung. Das einzelne, sichtbare Eiweißkörnehen ist jedenfalls schon ein Konglomerat einer größeren Anzahl von Eiweißmolekiulen.

Auch in Glykogenlösungen sieht man zahllose Körnchen. Nach Zusatz von saccharifizierendem Enzym (Speichel) verschwinden diese, wie $R$ a e h 1 . m a $\mathbf{n} \mathbf{n}$ beobachtete, mit dem Grade der Verzuckerung mehr und mehr.

In reiner Zuckerlösung zeigt, ebensowenig wie in den Lösungen kristallisierter, einfacher Stoffe selbst von sehr hohem Molekulargewicht, das Ultramikroskop absolut nicht mehr als das gewöhnliche Mikroskop.

Alle diese Untersuchungen von Flüssigkeiten oder gefärbten Gläsern werden mit dem zuerst beschriebenen Apparat gemacht. Die zweite Einrichtung, die mit dem zentral abgeblendeten Objektiv, ist dagegen zum Studium gewöhnlicher, zwischen Objektträger und Deckglas montierter Präparate geeignet. Wir werden uns da zunächst mit der Frage zu beschäftigen haben, wie denn ein größeres, in mikroskopischen Dimensionen liegendes Objekt, sich bei dieser ultramikroskopischen Betrachtung verhält. Nehmen wir als einfachstes Objekt eine Fig. 4 kreisförmige Scheibe, etwa ein rotes Blutkörperchen, so ist von einer stärkeren VergröBerung durch das Ultramikroskop keine Rede. Wir wenden ja dieselben Linsen an wie sonst. Aber der Eindruck ist doch absolut anders. Statt einer einfachen, kreisförmigen Kontur sehen wir mehrere konzentrische Ringe, die Beugungsringe; die Konturen sind alle wie mit einer Rundschriftfeder gezogen, verdoppelt oder verdreifacht. Zwei sich fast berührende Kreise erscheinen im Ultramikroskop wie eine mehrfach konturierte 8-Figur, und andere, nur um weniges kompliziertere Objekte geben infolge der gegenseitigen Überdeckung der Beugungsringe unentwirrbare Figuren. Für solche Objekte schafft das Ultramikroskop nur Verwirrung, nicht Klarheit. Deshalb kann gar keine Rede davon sein, daß man durch das Ultramikroskop über die feinere Struktur der tierischen Zelle oder dgl. genaueren Aufschluß erhält als bisher; bisher gibt es nur wenige, auch mikroskopisch sichtbare Objekte, deren ultramikroskopisches Studium von Vorteil ist. Denkt man sich z. B. innerhalb eines Blutkörperchens einzelne, wenige, räumlich gut getrennte Körnchen (wie sie 
bei gewissen Erkrankungen des Blutes vorkommen), so werden wir diese ultramikroskopisch müheloser erkennen, weil sie infolge der sie umgebenden Beugungsringe unnatürlich groß erscheinen. Bleibt man sich dessen bewußt, $\mathrm{da} B$ und wie die Form der $\mathrm{Ob}$ jekte durch das Ultramikroskop entstellt wird, so ist es für ähnliche Objekte manchmal mit Vorteil zu verwenden. Doch das wird Sie als Chemiker weniger interessieren.

Noch ein anderes Gebiet will ich kurz streifen. Es wird Ihnen bekannt sein, daß eine große Reihe von Krankheiten von Mikroorganismen hervorgerufen wird, welche so klein sind, daß wir sie selbst mikroskopisch nicht wahrnehmen können; so klein, daß sie selbst die Poren eines Kieselgurfilters passieren, welche für Bakterien sonst völlig undurchlässig sind. Dahin gehört z. B. vor allem die Maulund Klauenseuche, wie aus den Untersuchungen von L ö f $f$ l e r hervorgeht, und das Gelbfieber. Anfangs hatte man gehofft, diese Lebewesen nun mit dem Ultramikroskop sehen zu können. Bisher ist das aber nicht möglich gewesen. Da man auf eine Erkennung der Form verzichten muß, so ist eine Unterscheidung etwaiger ultramikroskopischer Organismen von Eiweißkörnchen äußerst schwierig. Nicht einmal die etwaige Eigenbewegung ist mit Sicherheit als Unterscheidungsmerkmal zu verwerten, denn wir haben ja gesehen, daß auch leb. lose Eiweißkörnchen eine ziemlich lebhafte Eigenbewegung entfalten. Jedoch will ich mich mit der Erörterung dieser Schwierigkeit begnügen, weil dieses Kapitel nicht in Ihrem Interessenkreis liegt.

\section{Der Niedenfïhrsche Intensivbetrieb nach dem D. R. P. 140825 .}

\section{Von E. Hartmann und F. Benker.} (Eingeg. d. 14.|2. 1906.)

Der jüngste Nieden $\mathrm{f}$ ü h $\mathrm{r}$ sche Aufsatz (diese Z. 19, 61 [1906]) gibt uns Veranlassung, etwas näher auf die Erfahrungen einzugehen, welche man in der Fabrik der früheren Société Roubaisienne in Wattrelos b. Roubaix mit der Arbeitsweise nach dem D. R. P. 140825 gesammelt hat.

Schon in dem L ü t y schen Vortrage (diese Z. 18, 1253 [1905]) wurde diese Anlage besonders erwähnt, und es wurden aus den in diesem Vortrag angeführten Resultaten Schlüsse gezogen, die zu einer falschen Beurteilung gerade dieses Systems führen mußten. Wir unterließen es, in unserem jüngsten Aufsatz (diese Z. 19, 132 [1906]) auf diese Punkte näher einzugehen, da wir annahmen, daß $L$ ü t y vielleicht nicht genügend genug unterrichtet sein könnte. Da nun aber nach der jüngsten Veröffentlichung des Patentinhabers selbst, diese französische Anlage wieder als ein wich tiges Argument für die mit dem D. R. P. $140825 \mathrm{zu}$ erreichenden Resultate aufgeführt wird, so dürfte es im allgemeineren Interesse liegen, die uns über diese Fabrik zur Verfügung stehenden tatsächlichen R esultate näher zu beleuchten.

Wir sehen uns überdies persönlich hierzu veranlaßt, weil die $N$ i ed e $n \mathbf{f} \ddot{u} \mathbf{h}-\mathrm{L} \ddot{\mathrm{u}}$ t y schen
Veröffentlichungen den SchluB nahe legen können, daB die in Roubaix nach unseren Plänen errichtete und nachher von $\mathrm{N}$ i eden $\mathrm{füh}$ mit seinem. Patent ausgerïstete Anlage früher $\mathrm{un} \mathrm{be}$ friedigende Resultate ergeben hätte. Tatsache ist, daß das von $N$ i ed en $f \ddot{u} h r$ in seiner Abhandlung angeführte System v or den seitens der dortigen Direktion nut ihm angeknüpften geschäftlichen Verbindungen überhaupt nicht im $\mathrm{Be}$ trieb gewesen ist.

Es ist ausgeschlossen, daß sich die diesbezügl. Mitteilungen des Herrn $\mathbf{N}$ i e d e $\mathbf{n} \mathbf{f} \ddot{\mathrm{u}} \mathbf{h} \mathbf{r}$, wonach dieses System nur $6 \mathrm{~kg}$ geliefert haben soll, auf ein anderes dortiges und ursprünglich von Delp l a c e-Paris erbautes System beziehen, da Genannter die Querschnittsverhältnisse ausdrücklich mit $5,5 \mathrm{~m}$ Breite bei $10 \mathrm{~m}$ Höhe angibt, die diejenigen der von uns in Roubaix errichteten Neuanlage sind, welche später mit dem $\mathrm{N}$ i e d e $\mathrm{n} f \ddot{u} \mathrm{~h} \mathbf{r}$ schen Patent ausgerüstet wurde. Aber auch bezüglich dieses Delplacesshen Systems stimmen die von Niedenführ angeführten Angaben durchaus nicht. Dasselbe leistete in seiner ursprünglichen Ausführung bei $5600 \mathrm{cbm}$ Kammerraum 20-22 t 53er Säure per 24 Stunden, was $3,8-4 \mathrm{~kg}$ pro cbm entspricht. Wir rüsteten dann dieses System mit einem Ventilator und mit unserer Wasserzerstäubung aus, und zwar unter garantierter Erhöhung der bisherigen Leistung um $30 \%$, erzielten aber tatsächlich trotz der ungünstigen Querschnittsverhältnisse der Kammern bei $10 \mathrm{~m}$ Breite und $10 \mathrm{~m}$ Höhe eine Erhöhung der Leistung auf $35 \mathrm{t}=\mathbf{5 0} \%$, was $6,2 \mathrm{~kg}$ 53 er Säure ausmacht.

Später fügten wir den beiden ursprünglich von Delpla c e vorgesehenen Kammern des Systems, welche Einteilung wir im allgemeinen als eine unrationelle betrachten, noch eine kleine dritte Kammer von $600 \mathrm{cbm}$ Inhalt hinzu, wobei wir auf einen Gesamt-Kammerraum von $6200 \mathrm{cbm}$ kamen. Wir erzielten hierin, ohne etwas an den Ofen und an den sonstigen Einrichtungen zu ändern, und a us chli e $B \mathrm{lich}$ mit unserer Wasserzer'stäubung, eine Tagesleistung von $45-46$ t $53 \mathrm{er}$ Säure, was $7,2-7,4 \quad \mathrm{~kg}$. pro cbm ausmacht. Es ist uns danach unerklärlich, wie $\mathrm{N}$ i ed e $\mathrm{n} \mathbf{f} \ddot{\mathrm{u}} \mathrm{h}$ in seinem Aufsatze (S. 63 links) behaupten kann, daß das System nur $6 \mathrm{~kg}$ pro cbm erzeugen konnte; es gibt hierfür nur zwei Möglichkeiten, welche wir zur Vermeidung von Irrtïmern in unserem Interesse konstatieren möchten; entweder beziehen sich die $\mathbf{N}$ i eden f $\ddot{u} \mathrm{~h} \mathrm{r}$ schen Angaben auf das System einer ganz anderen Fabrik, oder aber sio sind falsch, wenn eben genannte Fabrik gemeint ist.

Eine ferner nicht den Tatsachen entspreohende

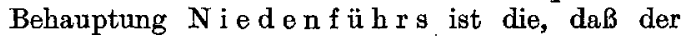
Querschnitt der von uns konstruierten Gay-Lussactürme zx eng bemessen gewesen soi. Wäro dieses der Fall gewesen, so wäre $\mathrm{N}$ i e d en $f$ ü $\mathrm{h} r$ bezüglich des Salpeterverbrauchs wohl nicht zu den Resultaten gekommen, die er für das System dort beansprucht, das von uns für eine Tagesleistung von $25-28 \mathrm{t} \mathrm{Kammersäure} \mathrm{von} 53^{\circ}$ Bé. konstruiert war.

Wir haben Herrn $\mathrm{N}$ ieden $\mathrm{f} \ddot{\mathrm{uh}} \mathrm{r}$ ersucht, diese nicht den Tatsachen entsprechenden An- 